\title{
Combustion Characteristics in Growth Chamber for Verneuil- grown Rutile Crystal
}

\author{
Xudong LIU ${ }^{1 \star}$, Lei WANG ${ }^{2}$, Huiyun YAN ${ }^{1}$, Xiaoguo BI ${ }^{3}$, Xudong SUN ${ }^{1,2}$ \\ 1 College of Environmental and Chemical Engineering, Dalian University, Dalian 116622 \\ 2 School of Materials Science and Engineering, Northeastern University, Shenyang 110819 \\ 3. School of Renewable of Energy, Shenyang Institute of Engineering, Shenyang 110136
}

*Corresponding Author: Xudong SUN, College of Environmental and Chemical Engineering, Dalian University, Dalian 116622, P. R. China. E-mail address: xdsun@mail.neu.edu.cn; Xudong LIU, College of Environmental and Chemical Engineering, Dalian University, Dalian 116622, P. R. China. E-mail address: liuxudong@dlu.edu.cn

\begin{abstract}
:
Combustion characteristics of three-tube burner in growth chamber for preparation of single crystal by the Verneuil method were investigated, and the effects of nozzle structure and flow rate on the surface temperature of molten cap were analyzed. The results showed that hydrogen flowed out from the nozzle diffused with inner and outer oxygen, and two flame produced in the center and near the wall of growth chamber. The surface temperature of molten cap were gradually reduced from the center outward. The temperature of molten cap decreased gradually with increasing the nozzle aperture of inner oxygen, and varied slightly with the diameter of hydrogen and outer oxygen nozzle. The temperature of molten cap decreased gradually with increasing the flow rate of inner and outer oxygen, while increased with the flow rate of hydrogen.
\end{abstract}

Keywords: rutile crystal; growth chamber; combustion; simulation

\section{Introduction}

The father of crystal fabrication technology is A. Verneuil with his flame-fusion growth method 1902. His principles of nucleation and growth control are adapted in most later growth methods from melt. The Verneuil method is one of the main methods for growing rutile single crystals ${ }^{[1-4]}$.

It was crucial for crystal growth to control the distribution of temperature and atmosphere in the growth chamber, especially near the crystal molten cap ${ }^{[5-10]}$. In the process of crystal growth, the too large radial temperature gradient of the growth chamber, especially near the crystal molten cap, is easy to cause the too low edge temperature of the flat molten cap, as shown in figure 1a, which makes the crystal size less likely to grow, or even shrink. While the radial temperature gradient in the vicinity of the molten cap is too small and the molten cap will be filled, as shown in figure $1 \mathrm{~b}$, which make the crystal molten cap is prone to overflow and get the crystal shown in figure 2 . The too large axial temperature gradient of the growth chamber shows that the cooling speed of crystal is too quickly, which causes the poor crystal integrity and the high brittleness, as shown in figure 3.
The quality of crystals not only depends on the temperature distribution, but also the oxidation or reduction atmosphere in the growth chamber. It is easy for $\mathrm{TiO}_{2}$ to lose oxygen, and not easy to crystallize in the reduction atmosphere of hydrogen-oxygen flame ${ }^{[11,12]}$. Therefore, the structure of burner was adjusted to obtain the temperature and atmosphere distribution in the growth chamber, which was favorable for the growth of rutile single crystals.

Flame fusion burners are customarily of the two-bube type with inner and outer tubes having tapered tips. The taper at the tip of the outer tube $\left(\mathrm{H}_{2}\right)$ has a tendency to constrict the flame pattern and enlarge the axial and radial temperature gradient. The taper at the tip of the inner tube constricts the $\mathrm{O}_{2}$ flow and the powder fall into a very narrow path so that both the oxygen flow and the powder strike the center of the growing crystal with high velocities sufficient to cause dishing out. In order to reduce the axial and radial temperature gradient, it is not enough to increase the diameter of center nozzle. The more flow rate of oxygen must be maintained to prevent the flareback when the diameter of center nozzle increases, which causes the 
excessive heat and local temperature for the crystals growth. So, the burner is constructed from three concentric tubes of different diameters. The center tube carrying the oxygen and feed material is of stainless steel. The intermediate and outer tubes carry the hydrogen and oxygen respectively. A water-cooled sleeve is placed over the outer tube of the burner. By adjusting the ratio of hydrogen and oxygen, the three-tube burner not only produce the rich oxygen atmosphere in the growth chamber, which inhibit the lack of oxygen in the growth progress of rutile single crystals, but also reduce the radial temperature gradient, which is conductive to the growth of crystal.

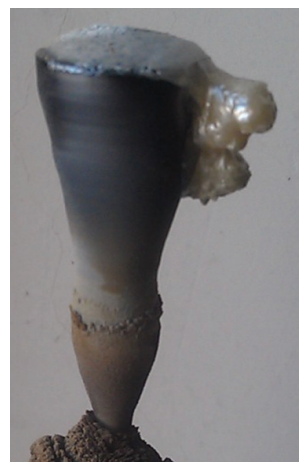

Figure 2. The overflow of rutile crystal

The structural perfection of rutile grown by various techniques has been related to the growth conditions and several characterization data on the flame-fusiongrown crystal have been published ${ }^{[13,14]}$. The patents and publications do not contain all the necessary data on the experimental conditions for growth of large rutile crystals, and there are few publications about numerical analysis of combustion characteristics in growth chamber for preparation of rutile crystals by the Verneuil method ${ }^{[15-17]}$. Therefore, a systematic numerical investigation on the flame-fusion growth process of rutile crystals was undertaken in order to evaluate the conditions required in the growth of crack-free crystals.

\section{Mathematical Model Formulation}

The structure of growth chamber is three-cone type, and

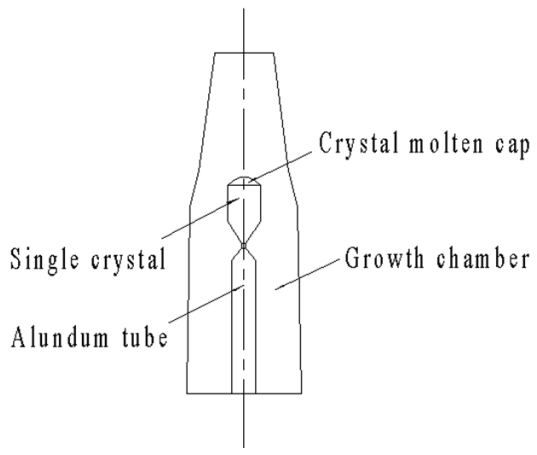

(a)

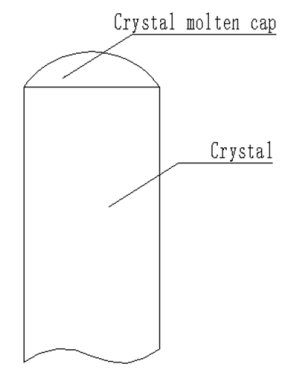

(a)

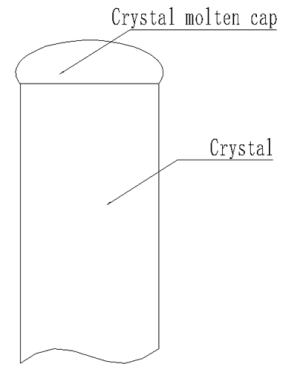

(b)

Figure 1. The oblate (a) and engorged (b) shape of crystal molten cap

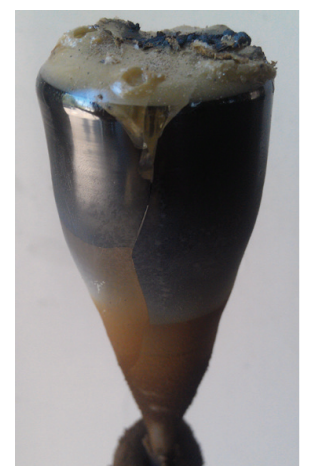

the height of growth chamber is $350 \mathrm{~mm}$. The diameters of growth chamber from upper to lower are $44 \mathrm{~mm}, 50 \mathrm{~mm}$, $60 \mathrm{~mm}$ and $70 \mathrm{~mm}$, respectively. The nozzle of burner is three concentric tubes structure. The diameter of center tube carrying the oxygen and $\mathrm{TiO}_{2}$ feed powder is $3 \mathrm{~mm}$. The inner and outer diameter of intermediate tube carrying the hydrogen is $22 \mathrm{~mm}$ and $24 \mathrm{~mm}$, and the inner and outer diameter of outer tube is $34 \mathrm{~mm}$ and $35 \mathrm{~mm}$. Considering the structure characteristics and symmetry of the growth chamber and the crystal, the schematic diagram, grid used for the computational models and structure size of growth chamber were determined as shown in figure 4 , in which the size of grid is $0.2 \mathrm{~mm}$ and the number of node is 171677 .

The temperature and atmosphere distribution in the growth chamber is mainly based on the combustion process of hydrogen and oxygen and the effect of flame

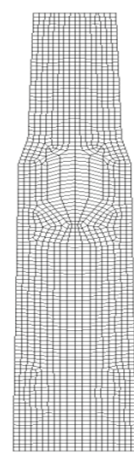

(b)

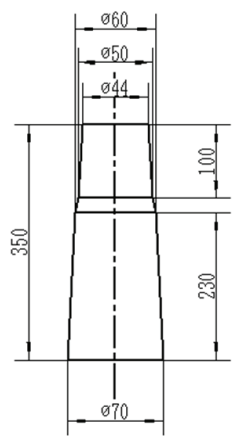

(c)

Figure 4. (a) schematic diagram, (b) grid and (c) structure size of growth chamber 
on the heat transfer of the crystal and corundum tube. In the combustion process, the coupling effect of chemical reaction, turbulent flow, convection and radiation heat transfer is analyzed. FLUENT software based on the finite volume and gradient algorithm is used to solve the CFD problems, such as convection and radiation heat transfer and combustion reaction, etc. The differential equations describing the combustion reaction, fluid flow and heat transfer of oxygen and hydrogen in the growth chamber are mainly included mass, momentum and energy conservation equations and heat transfer differential equation, and the mass conservation equation is given by ${ }^{[18]}$

$\frac{1}{r} \frac{\partial}{\partial r}\left(r \rho v_{r}\right)+\frac{\partial}{\partial x}\left(\rho v_{x}\right)=0$

Where $\rho$ is density, and $v_{r}$ and $v_{x}$ are velocity vector.

The momentum conservation equation is given by

$\frac{\partial\left(r \rho v_{x} v_{x}\right)}{\partial x}+\frac{\partial\left(r \rho v_{x} v_{r}\right)}{\partial r}=\frac{\partial\left(r \tau_{x}\right)}{\partial r}+r \frac{\partial \tau_{x}}{\partial x}-r \frac{\partial p}{\partial r}+\rho g_{x} r$

$\frac{\partial\left(r \rho v_{r} v_{x}\right)}{\partial x}+\frac{\partial\left(r \rho v_{r} v_{r}\right)}{\partial r}=\frac{\partial\left(r \tau_{r}\right)}{\partial r}+r \frac{\partial \tau_{x}}{\partial x}-r \frac{\partial p}{\partial r}$

Where $\mathrm{p}$ is pressure and $\tau_{i j}$ is stress tensor, which is defined as

$\tau_{x x}=\mu\left[2 \frac{\partial v_{x}}{\partial x}-\frac{2}{3}(\nabla \cdot V)\right]$

$\tau_{r r}=\mu\left[2 \frac{\partial v_{r}}{\partial r}-\frac{2}{3}(\nabla \cdot V)\right]$

$\tau_{r x}=\mu\left[\frac{\partial v_{x}}{\partial r}+\frac{\partial v_{r}}{\partial x}\right]$

Where $\mu$ is dynamic viscosity.

The standard $\mathrm{k}-\varepsilon$ model is determined for turbulent flow, and the equations of turbulent kinetic energy and dissipation rate are given by

$\frac{1}{r} \frac{\partial}{\partial r}\left[\left(\mu+\frac{\mu_{t}}{\sigma_{k}}\right) \frac{\partial(k)}{\partial r}\right]+\frac{\partial}{\partial x}\left[\left(\mu+\frac{\mu_{t}}{\sigma_{k}}\right) \frac{\partial k}{\partial x}\right]+G_{k}+G_{b}-\rho-Y_{M}=0$
$\frac{1}{r} \frac{\partial}{\partial r}\left[\left(\mu+\frac{\mu_{t}}{\sigma_{k}}\right) \frac{\partial(r \varepsilon)}{\partial r}\right]+\frac{\partial}{\partial x}\left[\left(\mu+\frac{\mu_{t}}{\sigma_{k}}\right) \frac{\partial \varepsilon}{\partial x}\right]+C_{1 \delta} \frac{\varepsilon}{k}\left(G_{k}+C_{3 \varepsilon} G_{b}\right)-C_{2 \varepsilon} \rho \frac{\varepsilon^{2}}{k}=0$

Where $\mu_{t}$ is turbulent viscosity, and $G_{k}$ and $G_{b}$ are turbulent kinetic energy generated by the average velocity gradient and buoyancy, respectively.

The energy conservation equation is given by

$\frac{1}{r} \frac{\partial}{\partial x}\left(r \rho v_{x} \int c_{p} d T\right)+\frac{1}{r} \frac{\partial}{\partial r}\left(r \rho v_{r} \int c_{p} d T\right)-\frac{1}{r} \frac{\partial}{\partial r}\left(r \rho D \frac{\partial \int c_{p} d T}{\partial r}\right)=-\sum_{j^{\prime}} h_{f^{\prime}} J_{j^{\prime}}$

Where $J_{j}$ is the diffusion flux of component $\mathrm{j}$.

The combustion reaction rate of oxygen and hydrogen is very fast, and the turbulent mixing of oxygen and hydrogen is much slower than the combustion process. As a result, the total chemical reaction rate is controlled by turbulent mixing, which can be determined as Magnussen and Hjertager vortex dissipation model, and expressed as

$R_{i, r}=v_{i, r}^{\prime} M_{w, i} A B \rho \frac{\varepsilon}{k} \frac{\sum_{P} Y_{P}}{\sum_{j}^{N} v_{j, r}^{\prime \prime} M_{w, j}}$

Where $\mathrm{Y}_{\mathrm{P}}$ is mass percentage of product $\mathrm{P}, \mathrm{A}$ and $\mathrm{B}$ are empirical constants, which determined as 4 and 0.5 respectively.

Heat transfer differential equation is given by $\frac{1}{r} \frac{\partial}{\partial r}\left(k \frac{\partial t}{\partial r}\right)+\frac{\partial}{\partial x}\left(k \frac{\partial t}{\partial x}\right)=0$

The boundary conditions are determined as following:

(1) The inlets of oxygen and hydrogen are the volume flow rates, and the flow rates of inner and outer oxygen and hydrogen are $7.2 \mathrm{~L} / \mathrm{min}, 3.8 \mathrm{~L} / \mathrm{min}$ and $19 \mathrm{~L} / \mathrm{min}$, respectively.

(2) The heat flow in the upper part of growth chamber is small, and treated to be an adiabatic boundary.

(3) Because the heat transfer in the axial direction of the furnace is much less than that in the radial direction, it can be neglected, and the heat transfer in radial direction is determined by

$q_{2}=h_{\text {eff }}\left(T_{w}-T_{f}\right)$

Where $h_{e f f}$ is the effective heat transfer coefficient, including the convection and radiation heat transfer from the furnace wall to the environment.

(4) The pressure of outlet of the growth chamber is zero.

The density and special heat of rutile crystals were $4270 \mathrm{~kg} / \mathrm{m}^{3}, 812 \mathrm{~J} / \mathrm{kgK}$ respectively, and the thermal conductivity was the fuction of temperature, as shown in Talbe 1 . When the temperature is greater than the melting point, considering the influnce of the flow on heat tuansfer, the effective thermal conductivity was used to the heat truansfer in crystal, so the effective thermal conductivity is greater than the thermal conductivity of solid crystals.

Table 1. the thermal conductivity of rutile crystals

\begin{tabular}{ccccccc}
\hline $\mathrm{T}(\mathrm{K})$ & 300 & 600 & 1000 & 2000 & 2123 & 2500 \\
\hline$\lambda(\mathrm{W} / \mathrm{mK})$ & 10 & 9.8 & 5 & 4 & 3.5 & 60 \\
\hline
\end{tabular}

\section{Results and Discussions}

\subsection{Temperature and Component Distribution}

The temperature and distribution of gas components in the growth chamber not only affect the combustion reaction, but also determine the stable growth process and microscopic quality of rutile crystal. In the actual growth process of rutile single crystal, the flow rates of inner and outer oxygen and hydrogen were $7.2 \mathrm{~L} / \mathrm{min}, 3.8 \mathrm{~L} / \mathrm{min}$ and $19 \mathrm{~L} / \mathrm{min}$ respectively when the crystal diameter was $24 \mathrm{~mm}$. According to the above equations and the boundary conditions, the FLUENT software was used to calculate the distribution of gas components in the growth chamber.

The radial temperature and component distribution characteristics of hydrogen, oxygen and water vapor at different distance from the nozzle, such as $10 \mathrm{~mm}, 50 \mathrm{~mm}$ and $90 \mathrm{~mm}$, were analyzed quantitatively, and the results were shown in figure 5 . The inner oxygen was mainly concentrated within the distance $50 \mathrm{~mm}$ from the nozzle, while the outer oxygen was mainly concentrated within the distance $30 \mathrm{~mm}$ from the nozzle and the trend diffusing to the wall of growth chamber was more obvious, as shown in figure 6. However, the radial molar fraction distribution of inner oxygen at the distance $90 \mathrm{~mm}$ from nozzle was 
obviously different from the others position, and the inner oxygen flowed outward along the radial direction and reacted to hydrogen surrounding crystal when it reached the surface of the molten cap, which led to increasing the edge temperature of crystal and was easy to cause the overflow problem.

It was difficult for hydrogen to spread to the inner oxygen and outer oxygen in the vicinity of the nozzle due to the large diffusion resistance produced by the high partial pressure of oxygen, and the two reaction peaks generated in the interface of hydrogen and oxygen. The reaction peak of outer oxygen and hydrogen disappeared and the distribution of hydrogen was gradually inclined to uniformity in the wall direction with the increasing of the distance from the nozzle, which showed that the outer oxygen was reacted completely and the oxidation atmosphere was supplied by the inner oxygen in the growth chamber. The concentration and radial concentration gradient of hydrogen decreased gradually with increase of distance from the nozzle. The farther the distance was, the more the diffusion and mixing of oxygen and hydrogen were. The combustion reaction of hydrogen and oxygen occurred in the process of mutual diffusion, and the molar fraction of water vapor was maximal when the molar fraction of hydrogen was the same as the oxygen.
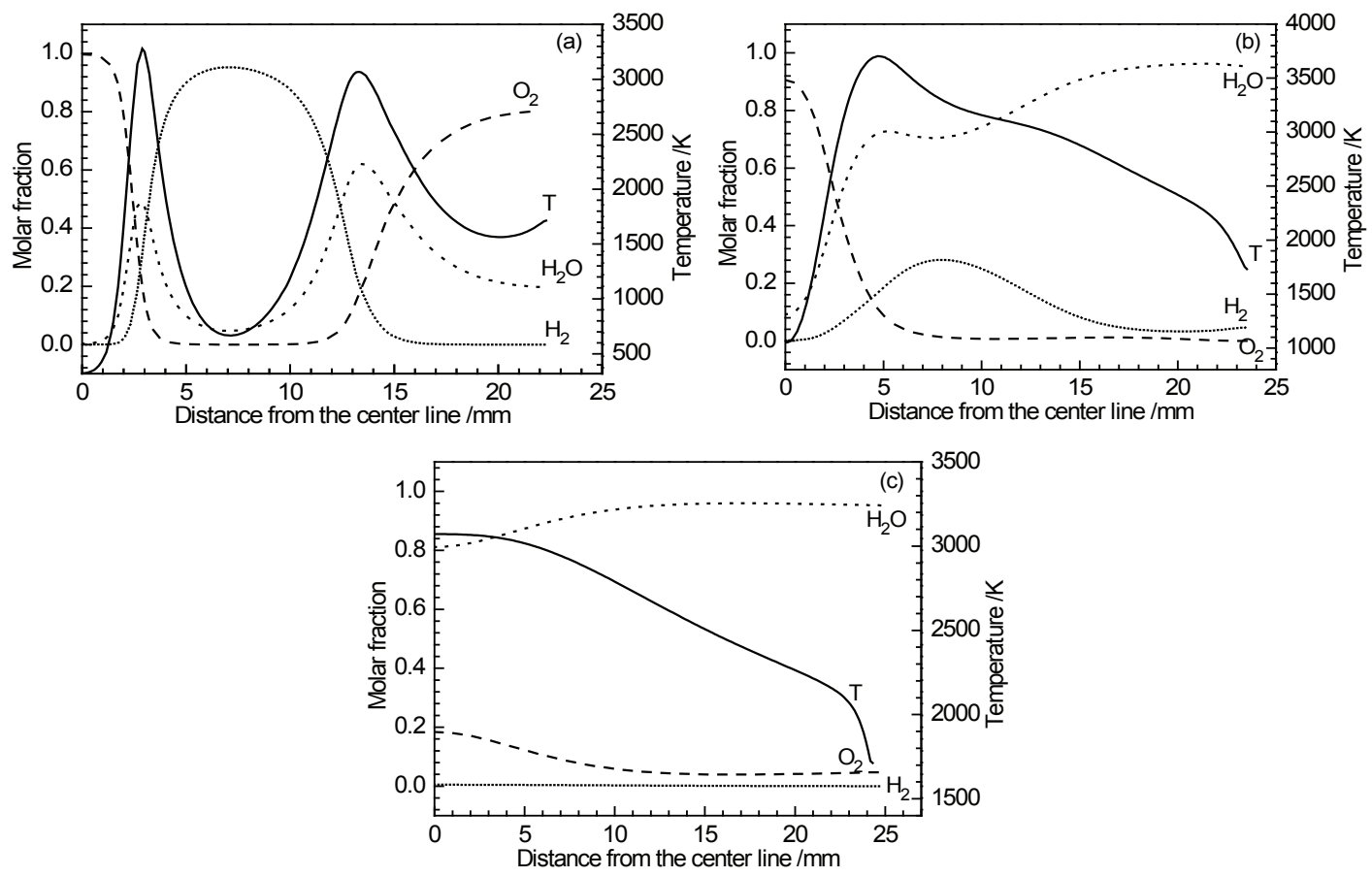

Figure 5. The temperature and molar fraction distribution of $\mathrm{H}_{2}, \mathrm{O}_{2}$, and $\mathrm{H}_{2} \mathrm{O}$ at different distance, 10mm (a), 50mm (b) and $90 \mathrm{~mm}$ (c) from the nozzle

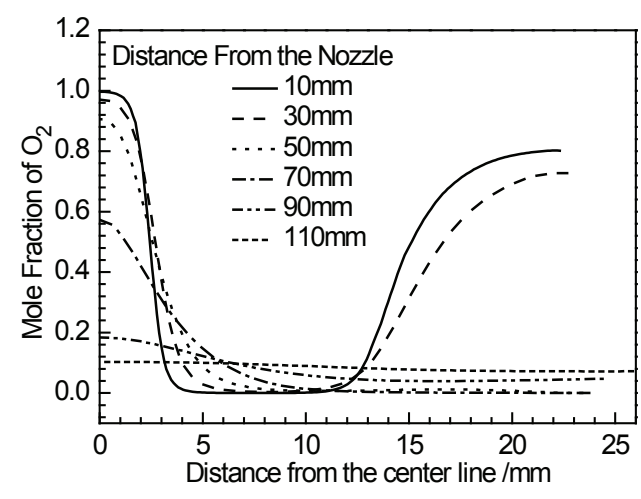

Figure 6. Radial oxygen distribution in growth chamber

The temperature distribution in the growth chamber was the most critical condition in the growth process of crystal, which determined whether the crystal was able to grow steadily and the final size and quality of crystal.
The hydrogen flowed out from the nozzle diffused with inner and outer oxygen, and two high temperature flame produced in the center and near the wall of growth chamber, as shown in figure 7 . Since the reaction rate of hydrogen and 
oxygen was very fast, the combustion process was mainly determined by the diffusion of hydrogen and oxygen. The mutual diffusion degree of outer oxygen and hydrogen was high and the combustion reaction was complete due to their similar blowing depth, which could be seen in figure 5 .

Whether the crystal was melting or crystallizing at the growth position was determined by the axial temperature in the growth chamber, and the radial temperature distribution determined the size of crystal. As can be seen from figure 5, there was two temperature peaks near the nozzle, and only one temperature peak far away from the nozzle, where the radial concentration of water vapor was maximal. The high temperature flame cooled from the reaction interface in the radial direction due to the heat lost from the wall of furnace. There was a temperature boundary layer near the wall, where the temperature was in a sharp decline. The radial temperature closed to the nozzle increased with decreasing the distance from the wall, which was more obvious at the position closer to the nozzle. This was because that the outer oxygen flow formed a vortex in the vicinity of the wall, and the high-temperature water vapor was involved in the vortex.

The growth state was determined by the temperature of molten cap of the growing crystal, which determined the superheat and viscosity of molten cap. If the superheat of molten cap was too large and the viscosity was too small, the molten cap was east to produce the large deformation when the greater pressure generated by airflow on it, which led to the overflow problem and the crystal growth failure, as shown in figure 2. While the molten cap was not easy to expand outward due to the too large viscosity, and the diameter of molten cap of the growing crystal decreased gradually. Sometimes the $\mathrm{TiO}_{2}$ powder blown into the molten crystal was not melted due to the lower melted superheat, which produced defects such as inclusions and crack in the crystal, as shown in figure 3. Therefore, it was very critical to analyze the temperature and oxygen distribution of the molten cap, as shown in figure 8 .

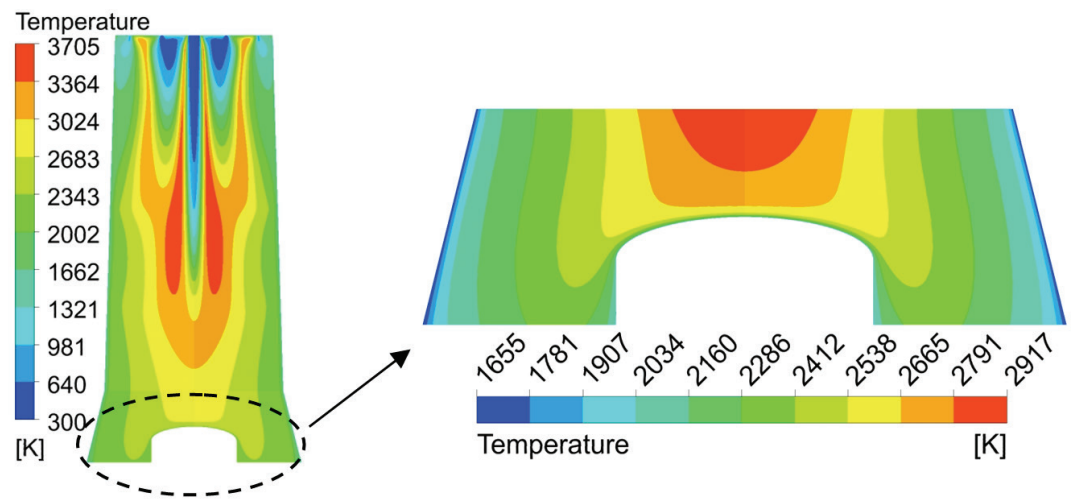

Figure 7. The temperature in the growth chamber

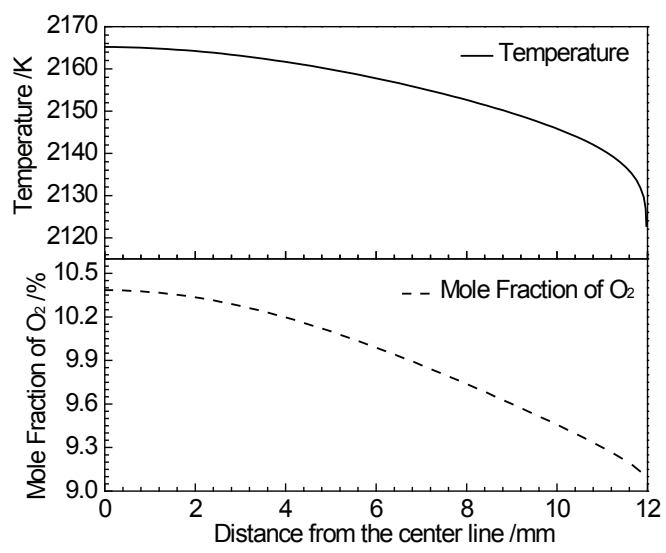

Figure 8. The distribution of radial temperature and mole fraction of oxygen in surface of molten cap

The surface temperature and mole fraction of oxygen of molten cap were gradually reduced from the center outward, and the gradient was small in the diameter range of less than $10 \mathrm{~mm}$. The magnitude of decreasing temperature increased with increasing the distance from the center line, and the maximum magnitude reached in the edge of the crystal.

\subsection{Effects of Nozzle Aperture}

The more and more flow rates of hydrogen and oxygen needed to meet the increasing diameter of crystal during the growth of rutile single crystal by the Verneuil method. If the nozzle aperture was too small, it was easy to form the turbulence in growth chamber when the flow rates of hydrogen and oxygen was larger, which caused the greater 
impact to the molten cap and the unstable crystal growth. If the nozzle aperture was too large, the larger flow rates of hydrogen and oxygen was required to maintain the molten crystal at the growth position, which led to the higher edge temperature of molten cap and caused the overflow of molten crystal. Therefore, it was critical to analyze the effects of nozzle aperture on temperature distributions

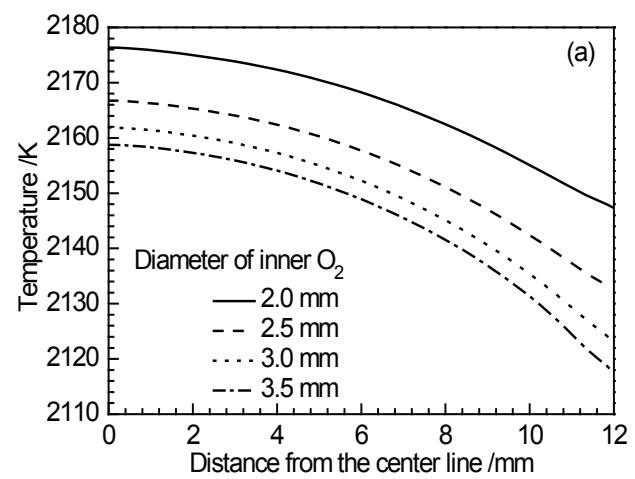

of the molten cap. The temperature distributions were calculated at the different nozzle apertures of inner oxygen, hydrogen and outer oxygen when the flow rates of inner and outer oxygen and hydrogen are $7.2 \mathrm{~L} / \mathrm{min}, 3.8 \mathrm{~L} / \mathrm{min}$ and $19 \mathrm{~L} / \mathrm{min}$ respectively, and the results were shown in figure 9.

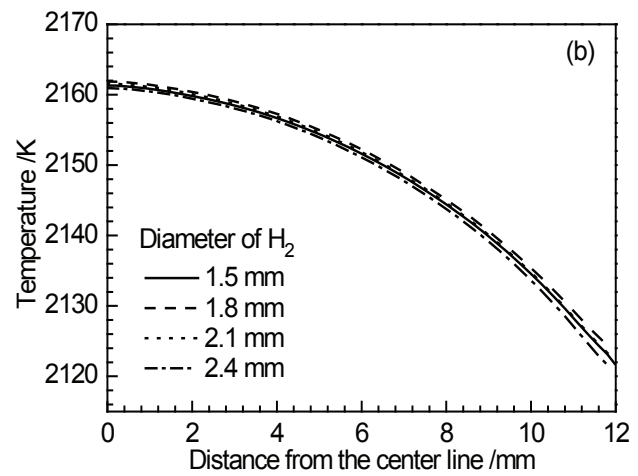

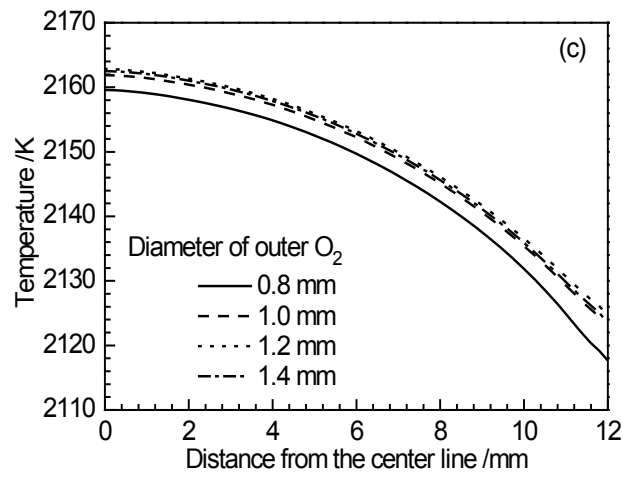

Figure 9. Effects of diameter of inner oxygen (a), hydrogen (b) and outer oxygen (c) on temperature in molten cap

The effects of nozzle aperture on the temperature of molten cap could be seen in figure 9. The temperature decreased gradually with increasing the nozzle aperture of inner oxygen, and the changing trend was more obvious in the smaller nozzle aperture. This was because that the hydrogen distribution in the growth chamber was essentially invariant when the apertures and the flow rates of hydrogen and outer oxygen were unchanged. The blowing depth of inner oxygen decreased and the diameter of oxygen flow increased with increasing the diameter of inner oxygen nozzle, which meant that the diameter of high-temperature zone of flame increased and the length decreased. So the center zone temperature of molten cap decreased with increasing the nozzle aperture of inner oxygen.

Although, the blowing depth and diameter of hydrogen flow changed with increasing the diameter of hydrogen nozzle, the effects of hydrogen distribution on the combustion reaction in growth chamber were little due to the good thermal expansion and diffusion of hydrogen. So the surface temperature of molten cap varied slightly with increasing the diameter of hydrogen nozzle.

As shown in figure 9(c), the surface temperature of molten cap varied slightly with increasing the diameter of outer oxygen nozzle due to the small change of blowing depth, which effected slightly by the diameter of outer oxygen.

\subsection{Effects of Flow Rate}

The growing diameter of crystal needed the more amounts of hydrogen and oxygen. The relative flow rates of hydrogen and oxygen not only determined the oxidation or reduction atmosphere, more importantly, but also the temperature distribution in the growth chamber during the growth process of rutile single crystal by the Verneuil method. In order to analyze the effects of flow rates of hydrogen and oxygen on the surface temperature of molten cap and the others were constant. The results were shown in figure 10 .

As could be seen in figure 10(a), when the flow rate of hydrogen and outer oxygen were kept constant, the temperature of molten cap decreased gradually with increasing the flow rate of inner oxygen. This was because that the combustion heat depended mainly on the flow rate of hydrogen when the flow rate of oxygen was excessive in the growth chamber. Invariable flow rate of hydrogen meant that the blowing depth and distribution of hydrogen had little change. The velocity of inner oxygen flow increased 
with the increasing flow rate of inner oxygen, which led to the more inactive inner oxygen flow. The inactive inner oxygen flow reduced the temperature and increased the

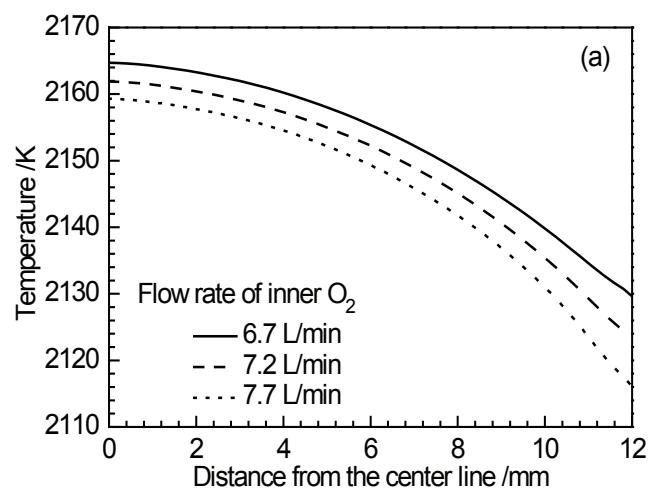

radial temperature gradient in the molten cap, which increased the internal thermal stress of crystal and reduced the crystal quality.

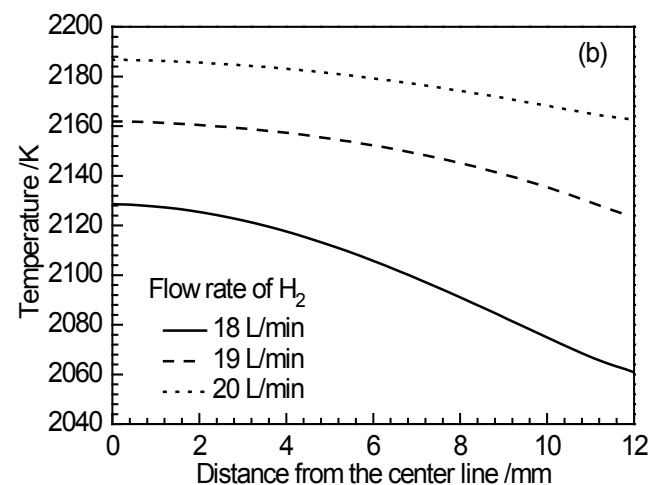

(c)

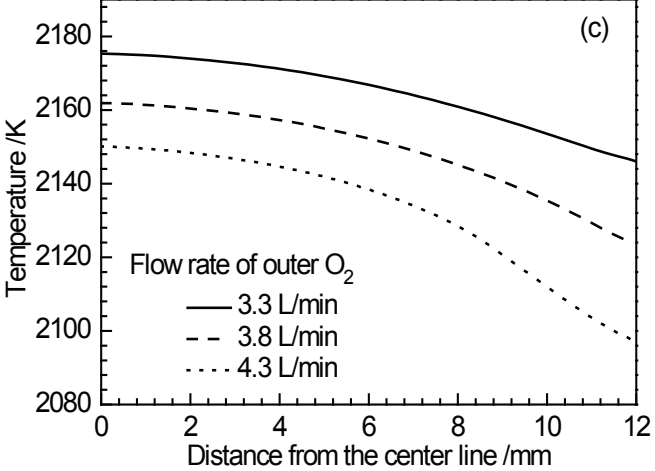

Figure 10. Effects of flow rate of inner oxygen (a), hydrogen (b) and outer oxygen (c) on temperature in molten cap

The surface temperature of molten cap increased obviously with the increasing flow rate of hydrogen. Theoretically, the total combustion reaction heat depended on the flow rate of hydrogen under the conditions of excessive oxygen flow in the growth chamber. The blowing depth of centric oxygen flow unchanged basically due to the same in inner oxygen flow when the flow rate of hydrogen changed. With the increasing flow rate of hydrogen, the blowing depth of hydrogen increased, which led to the more oxygen reacted with hydrogen and decreased the distance between the high temperature flame and the molten cap and increased the surface temperature of molten cap. The diffusion ability and diameter of hydrogen increased with the increasing flow rate of hydrogen, which led to the more diameter of high temperature zone. Therefore, the more flow rate of hydrogen need to meet the increasing crystal size in the process of rutile crystal growth, and increase the flow rate of hydrogen slowly in order to avoid the edge of the crystal temperature too high and cause overflow.

As could be seen in figure 10(c), when the flow rate of inner oxygen and hydrogen was kept constant, the temperature of molten cap decreased gradually with increasing the flow rate of outer oxygen. This was because that the combustion heat depended mainly on the flow rate of hydrogen when the flow rate of oxygen was excessive in the growth chamber. Invariable flow rate of hydrogen meant that the blowing depth and distribution of hydrogen had little change, the more hydrogen reacted with outer oxygen and the less hydrogen reacted with inner oxygen, so the surface temperature of molten cap decreased with the increasing flow rate of outer oxygen. The more increasement of outer oxygen flow was, the more inactive inner oxygen flow was, which reduced the temperature in molten cap.

\section{Conclusions}

The hydrogen flowed out from the nozzle diffused with inner and outer oxygen, and two high temperature flame produced in the center and near the wall of growth chamber. The surface temperature of molten cap were gradually reduced from the center outward. The temperature of molten cap decreased gradually with increasing the nozzle aperture of inner oxygen, and the changing trend was more obvious in the smaller nozzle aperture. While they varied slightly with the diameter of hydrogen and outer oxygen nozzle. The temperature of molten cap decreased gradually with increasing the flow rate of inner and outer oxygen, while increased with the flow rate of hydrogen.

Acknowledgements: This work was supported by the Projects of National Natural Science Foundation of China, No. 51872033 and No. 51732007, and by the Project of Dalian Natural Science Foundation, No. 2019J12GX044, and by the Project of Natural Science Foundation of Liaoning Province, No. 2019-ZD-0572. 


\section{References}

[1] Joseph A. Adamski. New Oxy-Hydrogen Burner for Flame Fusion[J].Journal of Applied Physics, 1965, 36(5):17841786

[2] Hans J. Scheel. Historical aspects of crystal growth technology[J]. Journal of Crystal Growth, 2000, 211: 1-12

[3] H. J. Scheel. Crystal growth technology CGT for energy: saving energy and renewable energy[J], Journal of Crystal Growth, 2005, 275:331-337

[4] Dr.-Ing. Hans J. Scheel, Dr. Peter Capper, Hans J. Scheel, et, al. Crystal Growth Technology: Chapter 16 FlameFusion (Verneuil) Growth of Oxides[M]. John Wiley \& Sons, Ltd, 2008, Nov. 25

[5] F. B. Khambatta, P. J. Gielisse and M. P. Wilson. Initial thermal model of the flame fusion crystal growth process[J].Journal of Crystal Growth, 1972, 13:710-717

[6] Floricica Barvinschia, Jean-Louis Santaillerb, Thierry Duffarb, Hervé Le Gal, Modelling of Verneuil process for the sapphire crystal growth[J], Journal of Crystal Growth, 1999, 198-199:239-245

[7] Shuichi Kawaminami, Keisuke Mochizuki, Shinobu Hashimoto, et, al. Coloration of Ti-doped sapphire grown by the Flame-Fusion Method[J]. Journal of Asian Ceramic Societies, 2013, 1(4): 362-367

[8] Xiao Guo Bi, Xu Dong Liu, Wei Niu. Flame-fusion growth of rutile single crystal[J].Advanced Materials Research, 2012, 535-537: 2571-2575

[9] J. B. Donnet, H. Oulanti, T. Le Huu, M. Schmitt. Sythesis of large crystal diamond using combustion-flame method[J]. Carbon, 2006, 44(2):374-380

[10] H. Tang, H. Li and J. Xu, Advanced Topics on Crystal Growth[M]. Ed. by S. O. Ferreira, InTech, Croatia, 2013: 307-333

[11] K T Hwang, J H Pee, W S Cho, S H Eom, J K Lee, Effect of
Alumina Starting Materials on Spheroidization in Flame Fusion Method [J]. Materials Science Forum, 2010, 654656:2382-2385

[12] Xiaoguo BI, XIU Zhi-meng, MA Wei-min, Xudong SUN, Study on Growth of Rutile (TiO2) Single Crystals, Journal of Northeastern University( Natural Science), 2004, 25(10):977-979

[13] Xiaoguo BI, XIU Zhi-meng, Xudong SUN, ZHAO Hongsheng, CAO Zhong-jie, GUO Guo-you, XIAO Ji-tian, Experimental Study on Growth Conditions of Big- bulk Rutile Crystal[J], Journal of Synthetic Crystals, 2004, 33(2):244-249

[14] S H Eom, J H Pee, J K Lee, K T Hwang, W S Cho, Effects of Chemical Composition and Particle Size of Starting Aluminum Source on the Spheroidization in the Flame Fusion Process [J]. Journal of Korean Powder Metallurgy Institute, 2009, 16(6):431-437.

[15] LIU Xudong, BI Xiaoguo, TANG Jian, NIU Wei, DONG Yingnan, Study on combustion characteristic in growth chamber for preparation of single crystal with flame fusion method[J], Chinese Journal of Materials Research, 2015, 29(5):394-400

[16] LIU Xudong, BI Xiaoguo, TANG Jian, CAl Yunping, SUN Xudong, Numerical Simulation of Temperature Distribution in Growth Chamber for Preparation of Rutile Single Crystal with Flame Fusion Method[J], Journal of Synthetic Crystals, 2016, 1: 138-145

[17] LIU Xudong, BI Xiaoguo, TANG Jian, NIU Wei, SUN Xudong, Numerical simulation of the influence of growth parameters on the temperature distribution in growth chamber[J], Journal of the Chinese Ceramic Society, 2016, 44(5): 1-7

[18] Stephen R Turns. An Introduction to Combustion Concept and Applications[M]. Tsinghua university press, 2015 